\section{Where do you think you're going? Part I and Part II}

\section{Libby Kemkaran-Thompson}

Our direction in life is not determined by the goals we set, as just setting the goal is in no way an indication that you will actually get there. It is more about how we react to the limits we put on ourselves and our belief patterns around pain and pleasure which form our behaviours. It is impossible to work genuinely towards a goal we feel is impossible or is actively painful; the old adage of 'if you think you can't, you're right' has never been more true as our lives get busier and our jobs take us into a place where we spend more hours at work than we do in the house we are working so hard to pay for. Any goal we commit to usually has to squeeze into the tiny space left between finishing work and falling into bed exhausted.

So how do we get traction and begin to move towards those things we want which might appear out of our reach, too hard, too much work, or just blocked by someone else's attitude? It is not a question of 'well just get up an hour earlier'; we need to be clever about managing our energy, our commitments and our attitude rather than just parroting old-fashioned timemanagement principles. There is a better way, and it is more effective than simply creating more pain attached to those behaviours needed to reach the enactment of your goal.

\section{PART I - WHERE DO YOU THINK YOU'RE GOING?}

This workshop starts with the theory behind limiting beliefs and effective preparation for goal setting, then leads into how to actually set goals you can not only stick to, but enjoy the journey along the way. Growth mindset is explored and strategy for improving team mindset and building continuous improvement systems to help get your goals embedded into team culture. There are things that every individual can do, but more power to effect change comes with having better strategy - these lectures focus on the practical delivery of change into existing structure (and even achieving delivery through our perhaps, less than enthusiastic colleagues).

\section{PART II - WHAT DO YOU THINK YOU'RE DOING?}

Build a plan for your own life in this session, with templates given for 90-day, 1-year and 3-year plans. Take a 'Growth Mindset and Grit' test to see how likely you are to succeed! This is an interactive session rather than just the theory, with an opportunity to come out with workable plans that you will actually be inspired to achieve in the real world.

\section{KEY LEARNING OBJECTIVES}

- Understand the limiting beliefs that prevent you from achieving your goals over and over again, and the drivers that make you succeed

- Understand the power of effective goal setting and how to enjoy the journey along the way so that you do not just quit

- Understand what it will take to create lasting change in a team and in an individual and the power of habits that help rather than hinder your progress

\section{MULTIPLE CHOICE QUESTIONS}

1. What are the four Ds of action goals?
(A) Dither, distract, despair then don't do it after all as it's just too painful
(B) Delay, doubt, delegate to someone else then disappear before they realize what you've done
(C) Do it, deny you did it, denounce all participants and then do it all over again next week
(D) Delete, defer, delegate, just do it

2. What do goals need to be?
(A) Clearly defined outcome: 'What' qualified with exact 'How' and 'By whom, by when'
(B) So painful that you cringe every time you even think of them
(C) So unrealistic that you cannot even say them out loud
(D) Someone else's problem because I don't do that because I'm a vet and this is all just a bit New Age for me

3. What is excellence?
(A) Not an act but a habit, we are what we repeatedly do
(B) A bit intimidating and I think I'll settle for 'good enough'
(C) A high goal and one I don't think I can reach
(D) Something for other people 